\title{
Lapsing during Sleep Deprivation Is Associated with Distributed Changes in Brain Activation
}

\author{
Michael W. L. Chee, ${ }^{1}$ Jiat Chow Tan, ${ }^{1}$ Hui Zheng, ${ }^{1}$ Sarayu Parimal, ${ }^{1}$ Daniel H. Weissman, ${ }^{2}$ Vitali Zagorodnov, ${ }^{3}$ and \\ David F. Dinges ${ }^{4}$ \\ ${ }^{1}$ Cognitive Neuroscience Laboratory, Duke-National University of Singapore Graduate Medical School, Singapore 169611, Singapore, ${ }^{2}$ Department of \\ Psychology, University of Michigan, Ann Arbor, Michigan 48109, ${ }^{3}$ School of Computer Engineering, Nanyang Technological University, Singapore 639798, \\ Singapore, and ${ }^{4}$ Division of Sleep and Chronobiology, Department of Psychiatry, University of Pennsylvania School of Medicine, Philadelphia, Pennsylvania \\ 19104
}

Lapses of attention manifest as delayed behavioral responses to salient stimuli. Although they can occur even after a normal night's sleep, they are longer in duration and more frequent after sleep deprivation (SD). To identify changes in task-associated brain activation associated with lapses during SD, we performed functional magnetic resonance imaging during a visual, selective attention task and analyzed the correct responses in a trial-by-trial manner modeling the effects of response time. Separately, we compared the fastest $10 \%$ and slowest $10 \%$ of correct responses in each state. Both analyses concurred in finding that SD-related lapses differ from lapses of equivalent duration after a normal night's sleep by (1) reduced ability of frontal and parietal control regions to raise activation in response to lapses, (2) dramatically reduced visual sensory cortex activation, and (3) reduced thalamic activation during lapses that contrasted with elevated thalamic activation during nonlapse periods. Despite these differences, the fastest responses after normal sleep and after SD elicited comparable frontoparietal activation, suggesting that performing a task while sleep deprived involves periods of apparently normal neural activation interleaved with periods of depressed cognitive control, visual perceptual functions, and arousal. These findings reveal for the first time some of the neural consequences of the interaction between efforts to maintain wakefulness and processes that initiate involuntary sleep in sleep-deprived persons.

Key words: lapses; visual cortex; functional neuroimaging; cognitive control; attention; sleep deprivation

\section{Introduction}

Many serious industrial catastrophes, transportation accidents, and medical errors result from lapses of attention that occur when sleep-deprived individuals transiently fail to stay alert while fighting the tendency to fall asleep (Mitler et al., 1988; Dinges, 1995; Barger et al., 2006; Philip and Akerstedt, 2006). Such lapses can occur after adequate sleep [rested wakefulness (RW)] when one is engaged in monitoring tasks (Makeig and Inlow, 1993; Makeig and Jung, 1996; Van Orden et al., 2000; Peiris et al., 2006; Huang et al., 2008), but they increase markedly in frequency and duration the longer one is sleep deprived (Doran et al., 2001). The behavioral characteristics of lapses have been well characterized in sleep-deprived subjects (Lim and Dinges, 2008). However, our

\footnotetext{
Received Dec. 22, 2007; revised April 10, 2008; accepted April 10, 2008.

This work was funded by DSO National Laboratories, Singapore Grant DSOCL05141 (M.W.L.C.). D.F.D. was supported by Air Force Office of Scientific Research Grant FA9550-05-1-0293, the National Space Biomedical Research Institute through NASA Grant NCC 9-58, and National Institutes of Health (NIH) Grant NR04281. D.H.W. was supported by NIH/National Institute on Drug Abuse Grant 1R03DA021345-01. Annette Chen, Delise Chong, and William Rekshan III performed scans and were involved in data preprocessing. Vinod Venkatraman assisted with some of the earlier data analyses.

Correspondence should be addressed to Dr. Michael Chee, Cognitive Neuroscience Laboratory, Duke-National University of Singapore Graduate Medical School, 7 Hospital Drive, \#01-11, Block B, Singapore 169611, Singapore. E-mail:mchee@pacific.net.sg.

DOI:10.1523/JNEUROSCI.0733-08.2008

Copyright $\odot 2008$ Society for Neuroscience $\quad 0270-6474 / 08 / 285519-10 \$ 15.00 / 0$
}

understanding of the neural correlates of attentional lapses has been derived entirely from studies of well-rested individuals.

"Lapses" may refer to either incorrect responses to speeded trials or delayed responses to stimuli, but, regardless of how they are defined, lapses occurring after a normal night's sleep are thought to originate from transient disruptions of cognitive control processes that rely on the frontal lobes (Padilla et al., 2006; Weissman et al., 2006). For example, in EEG studies, lapses defined as incorrect responses are associated with decreased frontocentral event-related potentials before stimulus presentation (Padilla et al., 2006). Similarly, lapse severity, indexed by response slowing, correlates with the extent to which prefrontal regions exhibit reduced activity before stimulus presentation (Weissman et al., 2006).

We hypothesize, however, that a transient failure of cognitive control processes might not fully explain lapses after sleep deprivation (SD). This hypothesis is motivated in part by findings indicating decreased task-related blood oxygenation leveldependent (BOLD) signal (Chee and Choo, 2004; Drummond et al., 2004; Habeck et al., 2004; Lim et al., 2007) in brain regions other than those that are directly involved in cognitive control after SD, such as brain regions that support visual attention and sensory processing. Evoked potential studies further suggest deficits in sensory processing in the SD state (Oken et al., 2006). Thus, transient failures of cognitive control after a normal night's 
sleep may be accompanied by additional deficits after SD. Notably, all functional imaging studies to date, except one (Drummond et al., 2005), have pooled fast and slow responses while estimating brain activation, precluding the discovery of additional differences in neural activation that might only be evident during SD lapses. As such, studying changes in task-driven brain activation as a function of response time (RT), and across states, might prove informative.

We made three predictions as to how neural activation associated with lapses in SD might differ from lapses recorded after a normal night's sleep. First, in accordance with the biasedcompetition model of selective attention, lapses in non-sleepdeprived individuals trigger increases in frontoparietal activity that could compensate for less efficient perceptual processing (Weissman et al., 2006). We predicted that sleep deprivation would lead to attenuation of these transient, lapse-driven increases in peak signal. Second, because attenuation of extrastriate activation has been observed during tasks that engage visual processing during SD (Chee and Choo, 2004; Choo et al., 2005; Chee and Chuah, 2007), we anticipated additional lapse-related reduction of visual cortex activity during SD lapses. Third, given that $\mathrm{SD}$ can involve periods of reduced arousal relative to RW, we expected changes in subcortical activation (e.g., reticular and thalamic nuclei) during lapses in SD (Kinomura et al., 1996).

To test these predictions, we used a global/local selective attention task (see Fig. 1), in which participants identified either the global, large letters or the local, small letters of a hierarchical stimulus (Navon, 1977). We reasoned that, if our predictions were to prove correct, we would be able to differentiate neural responses related to lapses of comparable duration recorded in $\mathrm{SD}$ from those observed in RW.

\section{Materials and Methods}

Participants. Twenty-four right-handed, healthy adults (13 females; mean \pm SD age, $22.5 \pm 1.6$ years) participated in the experiment. Four participants were excluded from the final analyses because of excessive in-scanner motion ( $>3 \mathrm{~mm}$ across runs), whereas three were excluded because of an excessive number of lapses ( $>15 \%$ of total trials) during the sleep deprivation session, resulting in complete data for 17 subjects. In addition, in the data analysis, we only studied correct responses. This was to ensure that we did not analyze data that simply reflected participants falling asleep in scanner.

Participants were selected from respondents to a web-based questionnaire. They had to (1) be right-handed, (2) be between 18 and 35 years of age, (3) have habitually good sleeping habits (sleeping no less than $6.5 \mathrm{~h}$ each night in the month before the study), (4) score no more than 22 on the morningness-eveningness scale (Horne and Ostberg, 1976) (5) have no history of sleep disorders, and (6) have no history of any psychiatric or neurologic disorders. The sleeping habits of all participants were monitored throughout the 2 week duration of the study and only those whose actigraphy data indicated habitually good sleep (i.e., they usually slept no later than 1:00 A.M. and woke no later than 9:00 A.M.) were eligible for brain imaging.

Study protocol. Participants visited the laboratory three times. They first attended a briefing session during which the experimental procedure was explained to them and practiced. At the end of this session, each participant was given an actigraph to monitor sleep patterns throughout the study. The second and third visits involved participating in the functional magnetic resonance imaging (fMRI) experiment. The first scanning session took place $\sim 1$ week after the initial visit. The order of the two sessions (RW and SD) was counterbalanced across the participants. RW and SD scan sessions were separated by at least 1 week to minimize the residual effects of sleep deprivation on cognition. RW sessions commenced at 8:00 A.M. In SD sessions, participants were monitored in the laboratory from 7:00 P.M. onward, and scanning took place the next day at $\sim 6: 00$ A.M. We chose to test at these times because vehicular accidents

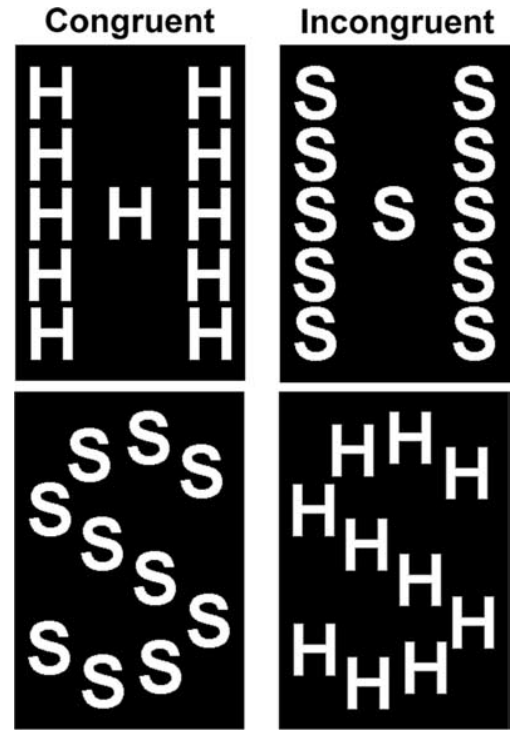

Figure 1. Task stimuli used in the experiment were single large, global letters ( $\mathrm{H}$ or $\mathrm{S}$ ) composed of several smaller local letters ( $\mathrm{H}$ or $\mathrm{S}$ ). The global letter and the local letters were either congruent (global $\mathrm{H}$ made up of local $\mathrm{Hs}$ and global $\mathrm{S}$ made up of local Ss) or incongruent (global $\mathrm{H}$ made up of local $\mathrm{Ss}$ and global $\mathrm{Ss}$ made up of local $\mathrm{Hs}$ ). A red fixation dot was displayed at the center of the screen throughout each run.

often peak at 6:00 A.M. after a night of sleep deprivation (Horne and Reyner, 1995). An important caveat to consider is that, although we attribute state-related differences in behavior and brain activation to sleep deprivation, a component of the observed effects may originate from the $2 \mathrm{~h}$ difference between the times at which we tested participants while they were in the RW and SD states.

During the SD session, participants were allowed to engage in nonstrenuous activities such as reading and watching videos. A research assistant observed participants throughout the night and prevented them from sleeping by verbal reminders. Participants were assessed with a psychomotor vigilance task sensitive to SD (Dinges et al., 1997; Doran et al., 2001) for $10 \mathrm{~min}$ every hour. Vigorous physical activity before the scans was not permitted. All participants indicated that they did not smoke or consume any medications, stimulants, caffeine, or alcohol for at least $24 \mathrm{~h}$ before scanning.

Experimental task. The task stimuli were single large, global letters $(\mathrm{H}$ or S; $\left.3.3^{\circ} \times 2.1^{\circ}\right)$ composed of several, smaller local letters $\left(\mathrm{H}\right.$ or $\mathrm{S} ; 0.6^{\circ} \times$ $0.4^{\circ}$ ) (Fig. 1) (Navon, 1977). The global letter and the local letters were either congruent (i.e., a global $\mathrm{H}$ made up of local Hs and global $\mathrm{S}$ made up of local Ss) or incongruent (i.e., a global $\mathrm{H}$ made up of local Ss and global Ss made up of local Hs).

In each trial, a single stimulus was presented centrally for $200 \mathrm{~ms}$, and participants identified the larger, global letter or the smaller, local letters by pressing one of two buttons. There were six runs of this task and 96 trials per run. Participants identified the global letter in three consecutive runs and the local letters in the remaining three. The order of the runs was counterbalanced across subjects and the two scanning sessions. With each run, there were equal numbers of congruent and incongruent trials. Congruent and incongruent trials were presented in a counterbalanced order that was predetermined for each participant such that there was an equal likelihood of a trial type appearing after every trial type in the design. The intertrial interval (ITI) ranged from 3 to $9 \mathrm{~s}$ and followed an exponential distribution that favored short ITIs. This distribution has been shown by simulation to be efficient in uncovering differences in signal strength elicited by various experimental conditions (Hagberg et al., 2001). To reduce the likelihood of nonlinear response summation (Soon et al., 2003), we increased the average ITI from 3.75 to $4.2 \mathrm{~s}$ compared with the original study (Weissman et al., 2006).

Imaging procedure and analysis. Stimuli were projected onto a screen at the back of the bore of the magnet using a liquid crystal display projector and viewed by participants through a mirror. Participants responded 

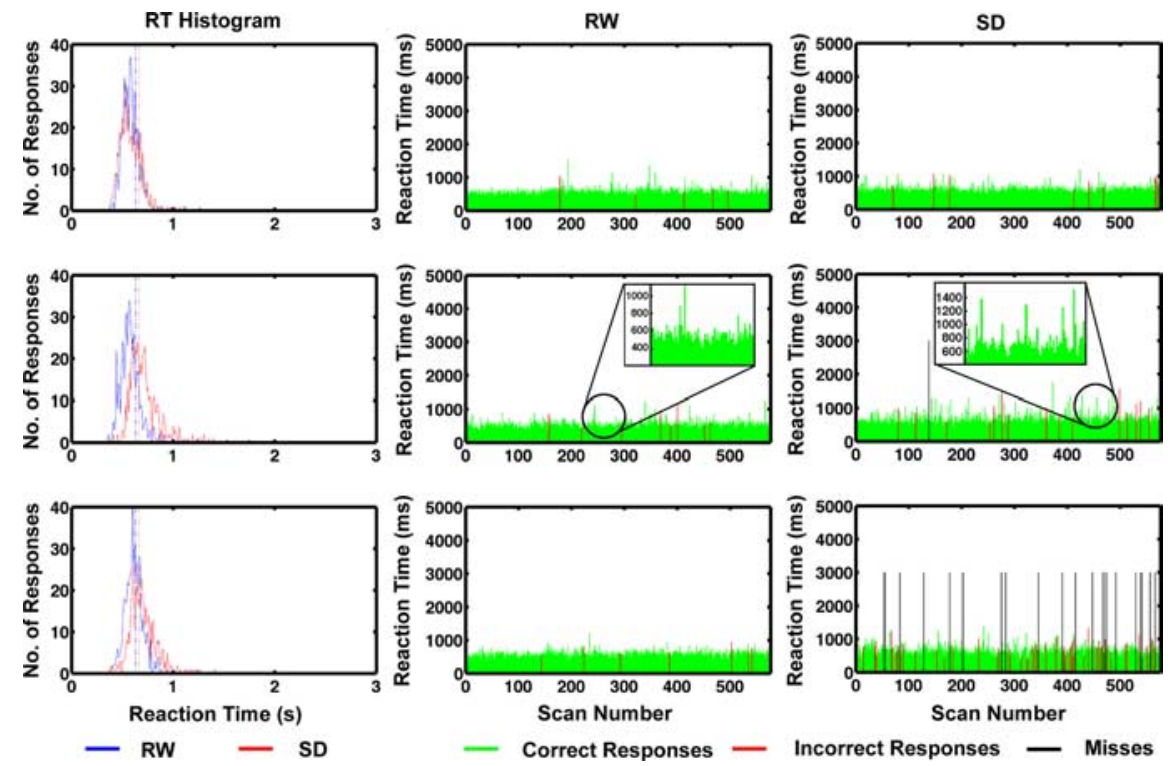

Figure 2. Illustrated are the RT histograms for correct responses and raster plots of responses associated with three representative subjects. Data were obtained during RW after a normal night's sleep or after 22-24 h of total SD. The top, middle, and bottom panels depict the distribution of responses and response patterns associated with an individual with few lapses, an individual with a moderate number of lapses, and an individual with a high number of lapses. The expanded inset illustrates the slower RT and greater trial-to-trial variation in RT when a subject was sleep deprived.

Table 1. Behavioral data after a normal night's sleep and after $24 \mathrm{~h}$ of sleep deprivation

\begin{tabular}{llllll}
\hline & RW & & & SD & \\
\cline { 2 - 3 } \cline { 6 - 6 } \cline { 6 - 6 } & Accuracy (\%) & Correct RT (ms) & & Accuracy (\%) & Correct RT (ms) \\
\hline Overall & $97.46(1.84)$ & $628(74)$ & & $94.1(4.1)$ & $660(63)$ \\
Congruent & $97.7(1.96)$ & $615(72)$ & & $95.57(3.01)$ & $646(62)$ \\
Incongruent & $97.22(1.89)$ & $640(78)$ & & $92.64(5.5)$ & $674(67)$ \\
\hline
\end{tabular}

Numbers in parentheses indicate standard deviation.

using a button box held in the right hand. Although we did not obtain simultaneous EEG, performance was continuously monitored (see Fig. 2) and participants were prompted to respond through the intercom system whenever they failed to respond to two consecutive trials. We used a bite bar and foam padding to reduce head motion. Images were acquired on a $3 \mathrm{~T}$ Allegra system (Siemens) using a gradient echo-planar imaging sequence [repetition time (TR), $1500 \mathrm{~ms}$; field of view, $192 \times$ $192 \mathrm{~mm}$; matrix size, $64 \times 64$ pixels]. Twenty-eight oblique axial slices $(4$ $\mathrm{mm}$ thick with a $0.4 \mathrm{~mm}$ interslice gap) parallel to the anterior commissure-posterior commissure line were acquired. High-resolution coplanar T1 anatomical images were also obtained. For the purpose of image display in Talairach space, an additional high-resolution anatomical reference image was acquired using a three-dimensional (3D) magnetization-prepared rapid-acquisition gradient echo sequence.

The functional images were processed using Brain Voyager QX version 1.8.6 (Brain Innovation) and custom routines written in Matlab (MathWorks). Intrasession image alignment to correct for motion across runs was performed. Interslice timing differences attributable to slice acquisition order were adjusted using linear interpolation. Gaussian filtering was applied in the spatial domain using a smoothing kernel of $8 \mathrm{~mm}$ full-width half-maximum. After linear trend removal, a high-pass filter of period $147 \mathrm{~s}$ was applied. Coplanar axial T1 images were used to register the functional dataset to the highresolution $3 \mathrm{D}$ image, and the resulting aligned dataset was transformed into Talairach space.

We used a finite impulse response model to estimate the average hemodynamic response across time that was evoked by each of our trial types. This model is a version of the general linear model in which the average hemodynamic response to each trial type is empirically derived without assuming a specific response shape (Miezin et al., 2000). For each trial type in the present study, we modeled two TRs ( $3 \mathrm{~s})$ before stimulus presentation and 12 TRs (18 s) after stimulus presentation. Only events that were associated with correct responses were considered in subsequent analyses.

Region of interest analyses. Task-related activation was determined by identifying voxels showing a significant difference in BOLD signal between the peak and post-peak undershoot phases of the average hemodynamic response to our stimuli in the RW state. Specifically, two time points from both the peak ( 4.5 and $6 \mathrm{~s}$ ) and the lowest (12 and $13.5 \mathrm{~s}$ ) points of this average, RT-indifferent $\mathrm{fMRI}$ response were contrasted. A random effects analysis of this contrast was conducted, and the resulting $t$ map was thresholded at $p<0.001$. The reasons for using the RW state as a reference point on which to base additional analyses have been discussed previously (Choo et al., 2005).

From this whole-brain analysis, four sets of significantly activated regions relevant to our predictions were chosen for region of interest (ROI) analyses on the basis of previous work using the same experimental paradigm (Weissman et al., 2006). These ROIs included (1) the medial frontal gyrus, (2) bilateral regions of the intraparietal sulcus, (3) bilateral regions of the lateral occipital cortex, and (4) the thalamus. Each ROI was centered at a local maximum in the task-related activation statistical map and had a minimum volume of 1 $\mathrm{cm}^{3}$. Of importance, the ROI analyses investigating state-related modulations of relationships between fMRI signal and response time were unbiased because they were unrelated to the contrast (i.e., all stimuli vs baseline) that was used to define the ROIs.

Two approaches to evaluating effects of response delay (lapsing) on brain activity. In evaluating the effects of response delay, two different approaches were used. The first approach was the trial-by-trial analysis method (Weissman et al., 2006), which has the advantage of providing an overview of how cortical activation changes with RT (see Figs. 3-5) and enables us to model brain activation associated with very infrequent slow responses that may be more behaviorally relevant than lesser RT delays. When using this approach, we restricted our analyses to events that were associated with correct responses to avoid including cortical events associated with sleep.

The general linear model that we used to conduct our trial-by-trial analyses included three main sets of predictors, which we estimated separately in the SD and RW states:

$$
h_{\mathrm{RT}}(t)=h_{0}(t)+(\mathrm{RT}-\overline{\mathrm{RT}}) h_{1}(t)+m(t)+\epsilon .
$$

The first set of predictors $\left[h_{0}(t)\right]$ modeled the average, RT-indifferent hemodynamic response to each trial type across time and used 14 finiteimpulse-response regressors as described previously. The second set of predictors $\left[h_{1}(t)\right]$ consisted of 14 regressors that modeled the contribution of RT to the average hemodynamic response at each time point of the average response of each trial type. For these RT regressors, the relative RT for each trial was the mean-subtracted RT for correct trials (Weissman et al., 2006). Of importance, the second set of predictors was completely orthogonal to the first set of predictors at each time point. Incorrect trials, trials associated with an omitted response, and trials associated with RTs $>3 \mathrm{~s}$ or $<0.3 \mathrm{~s}$ were modeled separately as misses $[m(t)]$ and were not analyzed further.

In summary, for each participant, multiple linear regression analyses yielded two sets of fMRI response curves per state. One set of response curves reflected the average cortical response to our stimuli across all RTs (see Fig. 3, left column), which we refer to as "task-related brain activation." Comparing task-related brain activation in the RW and SD states 
allowed us to determine whether, on average, different patterns of brain activation were present in the SD and RW states. The second set of response curves ("delay-related response") reflected the change in BOLD signal corresponding to a unit delay in responding relative to the mean RT for that state (see Fig. 3, right column).

To determine the overall effect of the task on cortical activation at each state, we summed the task-related activation curve, with the product of the RT delay and the second delay-related response curve:

$$
h_{\mathrm{LAPSE}}(t)=h_{0}(t)+0.5 h_{1}(t) .
$$

This combined curve was used to generate 3D plots that model how RT influences brain activation at each state and in various locations (see Fig. 4).

Although excellent for visualization, this trial-by-trial analysis of cortical response variation (Weissman et al., 2006) does not afford statistical analysis of state-related differences. As such, comparisons need to be performed at a particular RT. We therefore arbitrarily labeled responses that were at least 0.5 s longer than the mean RT lapses. This served to demarcate lapses as substantial response delays, differentiating them from lesser, possibly less significant, temporal deviations (Lim and Dinges, 2008). We analyzed between-state differences in brain activation at this particular time delay $(0.5 \mathrm{~s}+$ mean RT) so as to compare lapses of comparable duration across RW and SD.

To determine whether activation was significantly different across states (or across lapse and mean RT), we compared between-state activation magnitude at two time points: the peak of the response curve and one point after that. This was a planned comparison involving paired $t$ tests.

Despite the advantages of the trial-by-trial approach, a potential problem (supplemental data, available at www.jneurosci.org as supplemental material) is that it assumes that the magnitude of change in cortical response with RT is linear across different RTs, i.e., that the same neural processes underlie small and large delays in responding. Intuitively, most persons expect that there should be a difference in the neural underpinnings of lapses that lead to crashes and those that do not.

This motivated a second analysis approach: a direct comparison between activation associated with the fastest $10 \%$ and the slowest $10 \%$ of responses for each subject (Drummond et al., 2005).

For this analysis, we binned the fMRI responses associated with correct responses into three groups:

$h_{\mathrm{RT}}(t)=h_{\text {fastest } 10}(t)+h_{\text {slowest } 10}(t)+h_{\text {middle80 } 0}(t)+m(t)+\varepsilon$.

As before, we modeled cortical activation with 14 finite-impulse-response regressors for each state. However, instead of modeling RT delay directly, an additional three regressors were used: one coding for responses that were the fastest $10 \%$ for that subject $\left(h_{\text {fastest } 10}\right)$, one coding the slowest $10 \%$ of responses $\left(h_{\text {slowest10 }}\right)$, and one coding for the middle $80 \%\left(h_{\text {middle } 80}\right)$. The

$L$, Left; R, right; BA, Brodmann area.

Table 2. Talairach coordinates of regions that showed significant task-related activation $(n=17)$

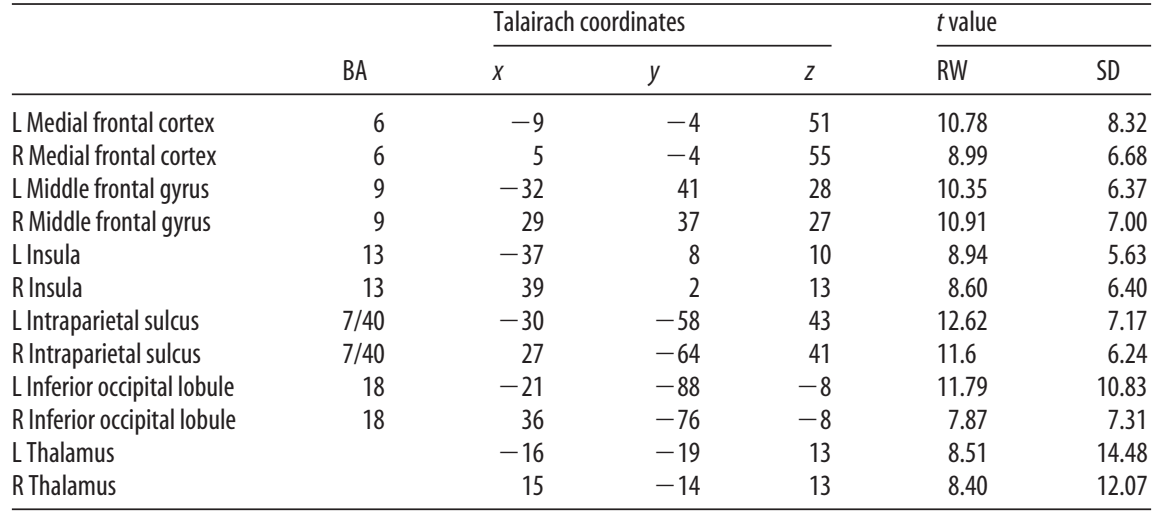

Mean Activity

Medial Frontal Cortex

$-6,-6,49$
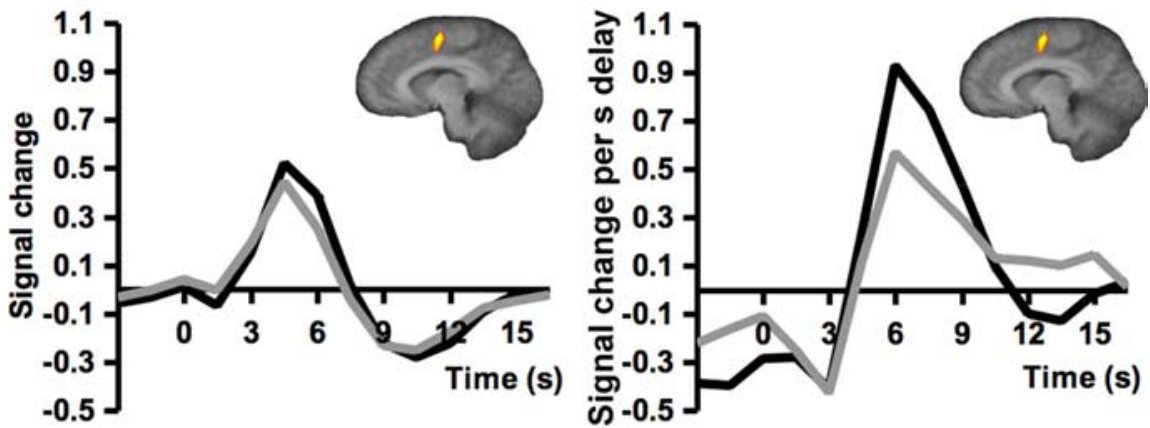

Intraparietal Sulcus

Left: $-28,-63,43$; Right: $29,-64,44$


RW

SD

Figure 3. Plots on the left half of the figure depict the average signal response after collapsing all correct responses regardless of RT. These reflect the mean task-related activation during RW after a normal night's sleep or SD. Plots on the right half of the figure depict the relationship between delay in RT and fMRI signal. Delayed responses were associated with a modest but state-indifferent reduction in peristimulus signal and larger, state-sensitive increases in peak signal in frontoparietal control regions. comparison of interest was between the fastest and slowest $10 \%$ of responses. This method of analysis considers the possibility that there might be an abrupt transition in the level of brain activation between behaviorally meaningful lapses and RTs only slightly longer than mean.

The disadvantage of this approach is that it yields a more conservative estimate of how cortical activation is modulated by RT. Furthermore, ignoring the middle range of RTs gives an incomplete appreciation of how brain activation is modulated by response delays. 




$p<0.005$; averaged left and right occipital, $t_{(16)}=4.73, p<0.001$ ) (Fig. 3).

Sleep deprivation does not modulate the degree to which reduction in peristimulus activity in frontal and parietal regions relates to lapses Previous studies suggest that reduced activity in frontal control regions around the time of stimulus onset predicts an upcoming attentional lapse (Padilla et al., 2006; Weissman et al., 2006). Accordingly, we found that cortical responses associated with lapses (Fig. 3) showed peristimulus signal reduction 1.5-3 s after stimulus onset in cognitive control regions (medial frontal cortex, $t_{(16)}=5.25, p<0.001$; bilateral intraparietal sulcus, $t_{(16)}=2.93$, $p<0.01)$. Interestingly, this signal reduction did not differ between the RW and the $\mathrm{SD}$ states. Moreover, this peristimulus reduction in signal was relatively small compared with the lapse-induced differences in peak signal that we observed in both states (compare peristimulus dip in Fig. 3, right column, with the same dip in Figs. 4, 5). Thus, in the ensuing analyses, we focus on the effects of lapses on peak signal magnitude.

Figure 4. 3D plots showing the results of trial-by-trial modeling of fMRI signal associated with RTs ranging from $0.2 \mathrm{~s}$ faster than the mean RT for a given individual, to 0.7 s slower than the mean RT. The signal time course at the mean RT is marked in green. $\boldsymbol{a}$, Medial frontal region; $\boldsymbol{b}$, intraparietal sulcus; $\boldsymbol{c}$, lateral occipital (extrastriate) cortex. Note that peak signal in the frontoparietal control regions increased with slower responses, albeit to a lesser extent during SD. In contrast, response slowing was associated with decrease in extrastriate peak signal during SD.

\section{Results}

Sleep deprivation results in slower, less accurate and more variable performance

Relative to RW, responses in SD were slower (660 vs $628 \mathrm{~ms} ; F=$ $12.17, p<0.005)$ and less accurate (94.1 vs $97.46 \% ; F_{(1,16)}=$ $19.00, p<0.001$ ) (Table 1). As expected, the distribution of response times to correct trials had a long right tail that was more pronounced after SD (Fig. 2). Also as expected (Van Dongen et al., 2004), SD was associated with increased intra-individual variability (intra-individual coefficient of variation $=\mathrm{SD}$ RT/mean RT) in response times $\left(0.25\right.$ vs $\left.0.20 ; F_{(1,16)}=8.55, p=0.01\right)$, as illustrated by the RT raster plot of the second of three illustrative participants (Fig. 2)

Consistent with previous findings (Weissman et al., 2006), incongruent trials elicited slower (657 vs $631 \mathrm{~ms} ; F_{(1,16)}=52.68$, $p<0.001)$ and less accurate $\left(94.94\right.$ vs $96.64 \% ; F_{(1,16)}=17.58, p<$ $0.005)$ responses compared with congruent trials. Furthermore, the difference in accuracy was magnified by SD (92.64 vs 95.57\%; $\left.F_{(1,16)}=5.64, p<0.05\right)$ without an accompanying speed-accuracy tradeoff $(r=0.11, p=0.69)$.

\section{Effects of sleep deprivation on average task-related activity}

Regions showing task-related activation are listed in Table 2. In accordance with previous findings indicating that SD modulates average task-related brain activation, we observed significantly reduced activation in SD compared with RW (medial frontal, $t_{(16)}$ $=3.32, p<0.005$; averaged left and right parietal, $t_{(16)}=3.38$,

\section{Lapses are associated with smaller increases of peak signal in frontoparietal control regions in the SD than in the RW state}

Higher task-related peak signal has been associated with slower and less accurate performance in working memory experiments (Honey et al., 2000; Manoach et al., 2000). In line with the hypothesis that lapses in RW stem from transient faltering of top-down control regions, lapses (i.e., trials with relatively slow responses) were associated with increased peak signal in various cognitive control regions (Figs. 3-5). Notably, although lapses increased peak signal in both states (relative to peak signal magnitude at the mean $\mathrm{RT}$ ), this signal elevation was more pronounced in the RW state (medial frontal, $t_{(16)}=5.14, p<0.001$; averaged left and right parietal, $\left.t_{(16)}=5.64, p<0.001\right)$ compared with the SD state (medial frontal, $t_{(16)}=3.31, p<0.005$; averaged left and right parietal, $t_{(16)}=3.53, p<0.005$; direct contrast between states; medial frontal, $t_{(16)}=2.11, p<0.05$; averaged left and right parietal sulcus, $\left.t_{(16)}=2.63, p<0.05\right)$.

Lapses are associated with larger reductions of visual cortical activity in the SD than in the RW state

The biased competition model of selective attention (Desimone and Duncan, 1995) has sometimes been used to explain how transient dysfunction of control and sensory regions could result in lapses. The model proposes that control regions determine resource allocation and guide the formation of high-quality perceptual representations of behaviorally relevant stimuli (Woldorff et al., 1993; Hopfinger et al., 2000; Kastner and Ungerleider, 2000). When these control regions falter, they could adversely affect visual cortex function, resulting in the formation of 
lower-quality percepts of behaviorally relevant stimuli (Weissman et al., 2006).

In the inferior occipital region, lapses in RW were not associated with either a larger or a smaller peak signal $\left(t_{(16)}=1.40, \mathrm{NS}\right)$, although there was an $\sim 1 \mathrm{TR}(1.5 \mathrm{~s})$ rightward shift in the average time-to-peak. In contrast, lapses in SD were associated with decreased extrastriate peak signal $\left(t_{(16)}=2.24, p<0.05\right)$ (Fig. 5). This reduction is consistent with other findings in extrastriate regions during tasks manipulating visual short-term memory and item load in sleep-deprived subjects (Chee and Chuah, 2007), and we will discuss these results more fully in Discussion.

Comparisons of brain activity for the fastest and slowest $10 \%$ of responses

To address concerns that linear modeling used in trial-by-trial analyses might not be appropriate for either very short or very prolonged RT, we reanalyzed the data by comparing statespecific cortical responses to an individual's fastest and slowest $10 \%$ of trials (Fig. 6). As with the linear model analysis, slower responses were associated with higher medial frontal and bilateral intraparietal sulcus peak signal in both states (RW: medial frontal, $t_{(16)}=3.6, p<0.005$; intraparietal sulcus, $t_{(16)}=3.61, p<$ 0.005 ; SD: medial frontal, $t_{(16)}=4.0, p<0.001$; intraparietal sulcus, $\left.t_{(16)}=3.71, p<0.005\right)$.

Between-state differences in peak signal magnitude involving the slowest $10 \%$ of trials were present in the bilateral parietal and occipital regions but not the medial frontal region (parietal, $t_{(16)}$ $=2.72, p<0.05$; occipital, $t_{(16)}=7.05, p<0.001$; medial frontal, $\left.t_{(16)}=1.37, \mathrm{NS}\right)$. Overall, then, the linear modeling used in our trial-by-trial analyses generated identical results except for the medial frontal area for slow responses, which showed no significant difference between RW and SD conditions.

Faster response times during SD elicited peak signals that were more similar to those observed in RW in frontoparietal control but not visual regions

State differences in peak signal within frontoparietal control regions were minimized when the fastest $10 \%$ of responses in each state were compared ( $\mathrm{t}<1$, NS, for the medial frontal and parietal ROI) (Fig. 6). This result is congruent with a voluntary engagement of wake-maintenance mechanisms during SD that results in periods of preserved responding sandwiched between several slower responses.

In keeping with our hypothesis that visual sensory processing is particularly impaired during $\mathrm{SD}$, we observed lower activation in the extrastriate visual cortex during SD even for the fastest responses $\left(t_{(16)}=2.69, p<0.05\right)$. We found this to be so in both the sorted responses (fastest 10\% of RT) (Fig. 6) and the modeled responses incorporating these faster response times (Fig. 4).

Thalamic responses showed state differences reflecting the interaction between arousal and attention

Arousal can be thought of as being brought to a state of readiness, whereas alertness refers to the state of readiness of vigilant attention to respond to external stimuli. Sleep-wake mechanisms modulating arousal derive primarily from subcortical structures, including brainstem, thalamic, and hypothalamic structures (Datta and Maclean, 2007). Because arousal is reduced after sleep deprivation, we might expect lower overall thalamic activation in the SD than in the RW state. Conversely, responding to a stimulus can temporarily increase attention and thalamic activation (Foucher et al., 2004) and may function to prevent lapses despite elevated sleep pressure (Kinomura et al., 1996). Consistent with
Medial Frontal Cortex $-6,-6,49$

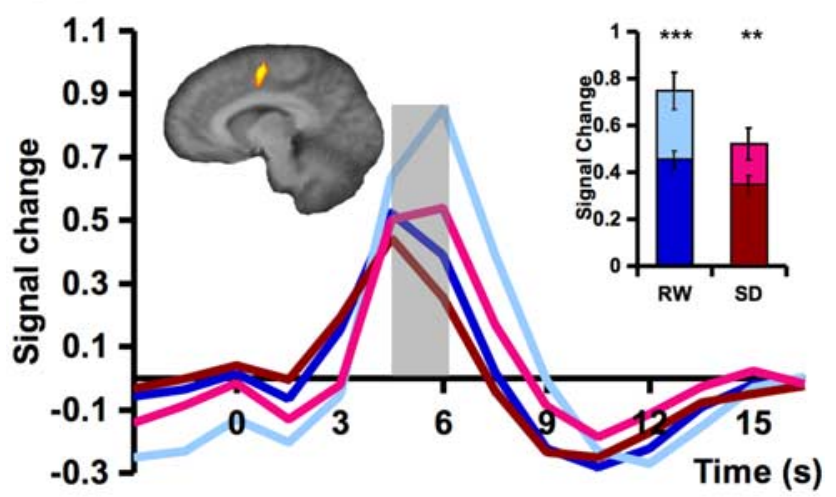

Intraparietal Sulcus

Left: $-28,-63,43$; Right: $29,-64,44$

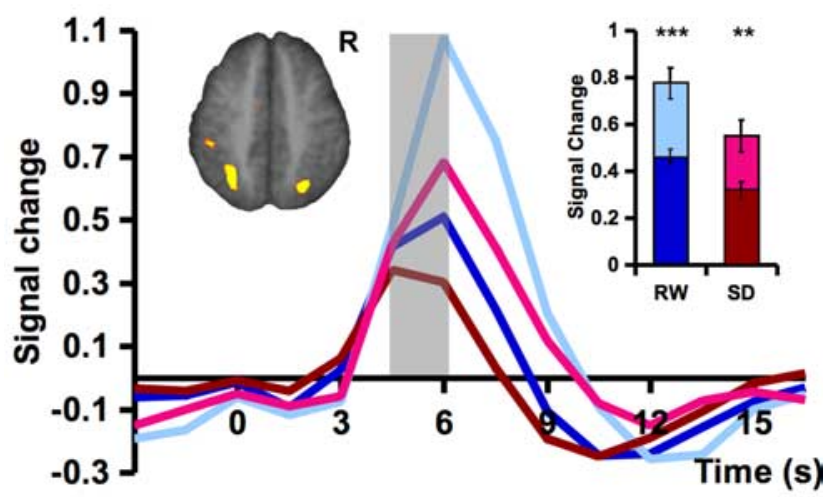

Inferior Occipital Cortex Left: $-35,-80,-5$; Right: $32,-77,-5$
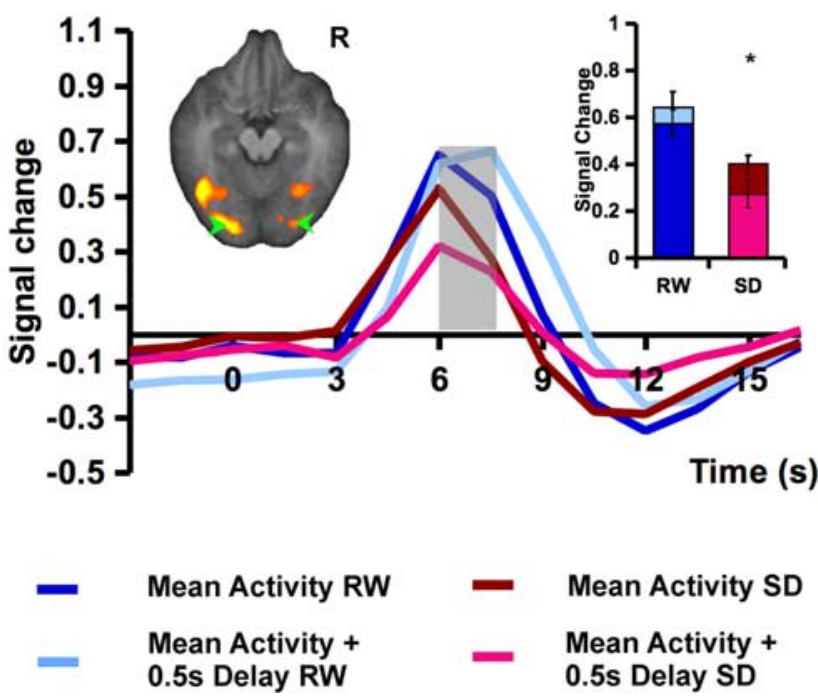

Figure 5. Task-related fMRI signal associated with responding at the average RT and during a lapse (modeled as a response 0.5 s longer than the average RT for that state). Lapses were associated with higher peak signal in the medial frontal cortex (top) and bilateral intraparietal sulcus (middle) in both RW and SD. In the occipital region, lapsing significantly reduced peak signal during SD (bottom) but did not significantly modulate the peak fMRI signal after RW, although there was a delay in the time-to-peak. Random effects analysis using a threshold of $p<0.001$ was used to detect task-related activation. Significant differences between peak signal associated with a lapse trial and the mean response for each state are marked with an asterisk. The shaded time points indicate those contrasted to assess significant state effects. The inset shows the mean peak signal associated with the time points under consideration. Error bars represent SEM. ${ }^{*} p<0.05,{ }^{* *} p<0.005,{ }^{* * *} p<0.001$. 


\section{Fastest 10\% RT}

\section{Medial Frontal Cortex}

$$
-6,-6,49
$$



\section{Intraparietal Sulcus}

Left: $-28,-63,43$; Right: 29, -64, 44

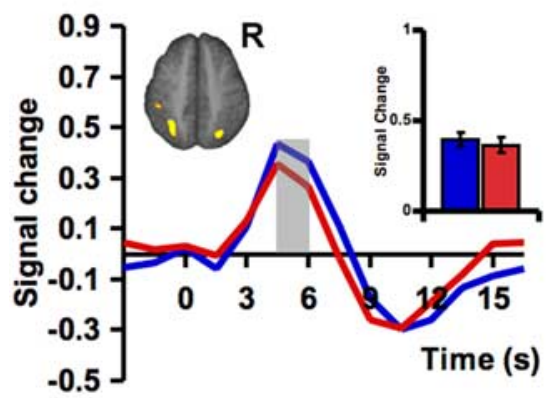

\section{Inferior Occipital Cortex}

Left: $-35,-80,-5$; Right: $32,-77,-5$


RW
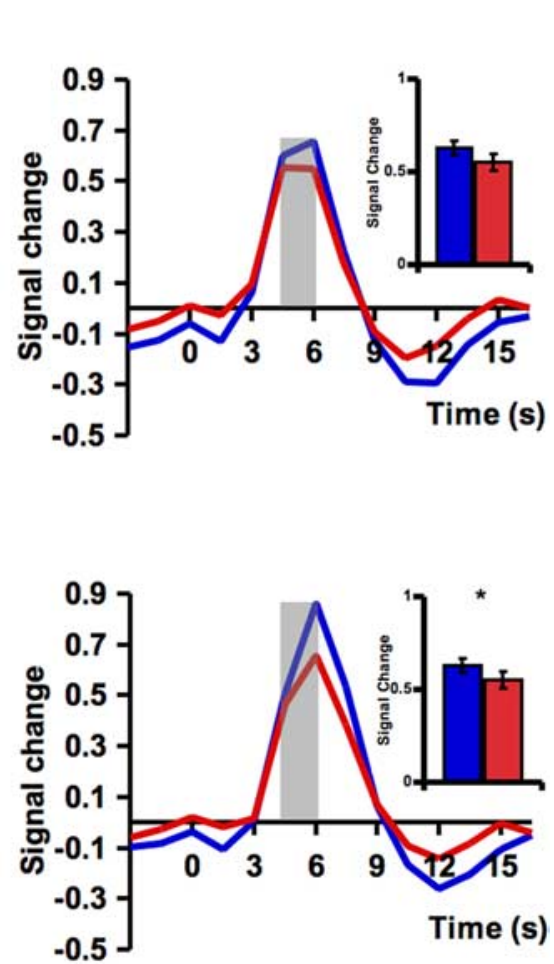

Slowest 10\% RT 

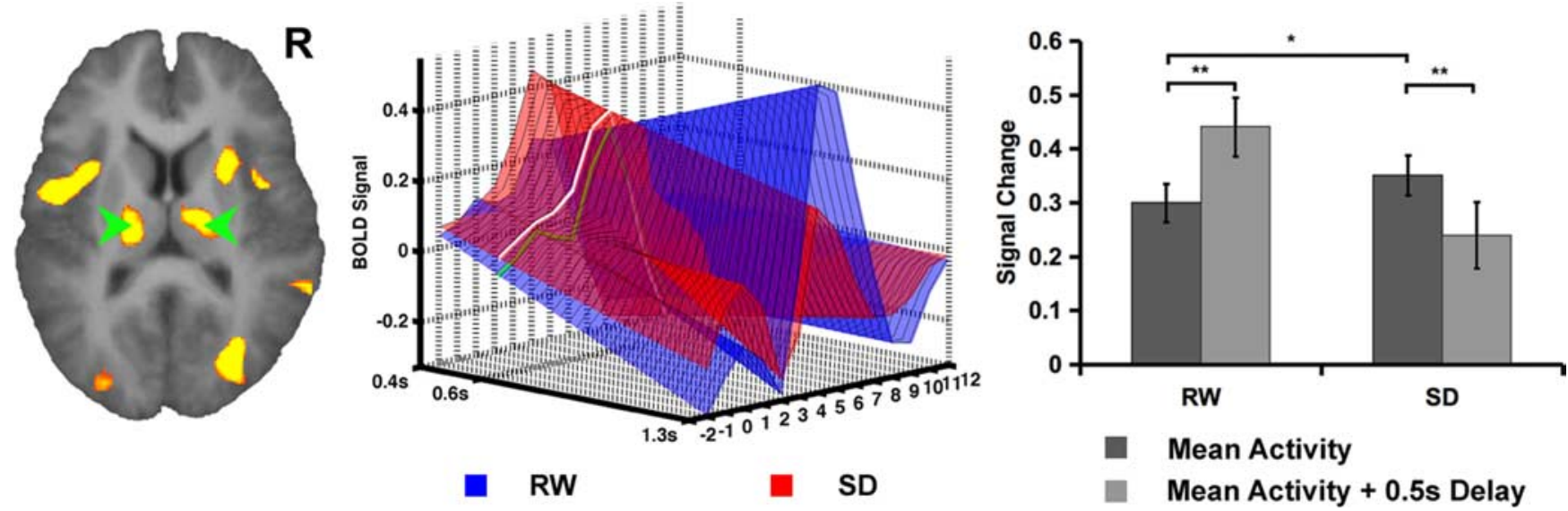

Figure 7. 3D plot showing the results of trial-by-trial modeling of fMRI signal in the thalamus. fMRI signal evolution for RTs ranging from $0.2 \mathrm{~s}$ faster than mean RT for a given individual to $0.7 \mathrm{~s}$ slower than mean RT are displayed. The signal time course at the mean RT is marked in green. Lapses in SD significantly attenuated task-related thalamic activation. This contrasted with RW in which lapses were associated with significantly greater peak fMRI signal. Error bars represent SEM. ${ }^{*} p<0.01,{ }^{* *} p<0.001$.

ory tasks (Chee and Choo, 2004). Interestingly, the expression of such state-dependent effects within the visual cortex may be contingent on the engagement of selective attention, because occipital activation in response to a passively viewed $8 \mathrm{~Hz}$ patternreversing checkerboard did not differ significantly between RW and SD (supplemental data, available at www.jneurosci.org as supplemental material).

SD-induced decline in attention/visual processing may be a consequence of reduced noradrenergic and cholinergic drive. Both neurotransmitters underlie the maintenance of wakefulness (Sarter et al., 2006) and visuospatial attention (Everitt and Robbins, 1997; Phillips et al., 2000). Reduced cholinergic drive accompanies prolonged wakefulness and increased sleepiness (Jones, 2005). Although no single neurotransmitter is necessary or sufficient to modulate sleep or wakefulness, we focus on the cholinergic system in the discussion because it strongly influences arousal, orienting and sustained attention, memory, as well as visual processing (Steriade and McCarley, 2005; Sarter et al., 2006; Bentley et al., 2008). Cholinergic neurons in the basal forebrain project extensively throughout the cerebral cortex and might be expected to have nonlocalized effects. However, pharmacological studies show a concentration of task-driven cognitive effects in parietal attention control regions (Davidson and Marrocco, 2000; Bentley et al., 2008) as well as in the extrastriate cortex (Furey et al., 2000), regions whose activation is modulated by $\mathrm{SD}$ in the present study. Acetylcholine can influence visual processing directly (Sato et al., 1987; Murphy and Sillito, 1991) or indirectly through top-down effects mediated by prefrontal (Everitt and Robbins, 1997) and parietal (Bentley et al., 2008) cortices. Additional experimentation is required to tease out the relative importance of these modulatory pathways in influencing performance.

Lapses during sleep deprivation are associated with deficits in top-down control

Transient disruptions of top-down control processes implemented via the frontal lobes are thought to underlie incorrect or slowed responses in selective attention tasks in RW subjects (Padilla et al., 2006; Weissman et al., 2006). In both states, we found that slower responses generated higher peak signal relative to faster responses, a finding congruent with several studies that compared activation in faster and slower individuals performing working memory tasks (Rypma and
D’Esposito, 1999; Honey et al., 2000; Rypma et al., 2006). Higher signal change in this context has been attributed to the engagement of neural circuits for a longer duration to complete the identical task, indicating lowered processing efficiency (Rypma et al., 2006).

Although slow responses in SD also elicited frontoparietal peak signal increase, an equivalent degree of lapsing elicited a smaller increase compared with RW. This finding could represent a change in problem-solving strategy during SD (Horne, 1988), but this possibility seems unlikely given that the task was relatively simple and highly practiced. Thus, in our view, the most probable explanation is that, in the SD state, there is a reduction in the number of neurons and/or the amount of time that frontoparietal neurons can be recruited to compensate for attentional lapses.

In advancing these hypotheses, we acknowledge that the relationship between cognitive efficiency and activation magnitude can appear to go in the opposite direction (Gray et al., 2003; Chee et al., 2004) from what we describe here depending on task and region (Rypma et al., 2006). For example, although our findings in the frontoparietal regions replicate those of a previous study using the same task (Weissman et al., 2006), they differ from those obtained using a different attention task (Drummond et al., 2005) and indicate the importance of considering the task used and the accompanying behavioral context before interpreting imaging findings.

\section{Wake-state instability: competing effects of top-down control} and sleep initiation

Performance variability after SD reflects the competing effects of maintaining wakefulness and falling asleep, resulting in what has been described as "wake-state instability" (Dorrian et al., 2005; Lim and Dinges, 2008). Such variability is clearly visible in the present study when considering trial-by-trial fluctuations in response time, especially in the SD state (Fig. 2). The present data are interesting in that lapses in the RW and in the SD states appear similar from a behavioral perspective but have appreciably different neural activation patterns.

There are multiple mechanisms involving networks of neurons within the brainstem, hypothalamus, and basal forebrain that could shift one from wake to sleep very quickly (Szymusiak et al., 2007), for example, the "sleep-on" cells in forebrain and hypothalamic regions (Uschakov et al., 2007). The ventral lateral 
preoptic nucleus has been proposed as a sleep switch that acts by inhibiting ascending excitatory systems that involve monoamine and cholinergic neurons (Saper et al., 2001). This sleeppromoting system has widespread and rapid effects on the cerebral cortex, possibly accounting for why we see effects of lapses in several cortical regions, although not necessarily in a uniform manner across the brain. This area becomes progressively less inhibited with prolonged wakefulness, possibly accounting for the greater frequency and longer duration of lapses when one is sleep deprived (Lim and Dinges, 2008). Opposing the onset of sleep initiation are top-down control systems that include the medial frontal and parietal cortices, whose effectiveness in sustaining goal-directed behavior is compromised after SD.

One consequence of the weakening ability of control regions to modulate attention during SD is the reduction of visual information flow from extrastriate regions, whose neurons respond to top-down influences (Kastner and Ungerleider, 2000; Corbetta and Shulman, 2002), to other cortical regions and the thalamus. Such attenuation of sensory processing may serve to insulate us from the environment to permit uninterrupted sleep (Livingstone and Hubel, 1981).

In summary, our findings suggest that lapses in the $\mathrm{SD}$ and the RW states differ in important ways. The most apparent of these is the attenuation of extrastriate activation that may precede a more widespread shutting down of responsiveness to the external environment. The consistency of this finding across two different methods of analysis is reassuring. However, a more thorough understanding of lapses during SD could benefit from teasing out the relative contributions of attenuated visual cortex function and the reduced top down control of attention as well as the neurotransmitter systems involved in the rapid transition from waking alertness to the putative microsleeps believed to underlie lapses in the sleep-deprived brain.

\section{References}

Barger LK, Ayas NT, Cade BE, Cronin JW, Rosner B, Speizer FE, Czeisler CA (2006) Impact of extended-duration shifts on medical errors, adverse events, and attentional failures. PLoS Med 3:e487.

Bentley P, Driver J, Dolan RJ (2008) Cholinesterase inhibition modulates visual and attentional brain responses in Alzheimer's disease and health. Brain 131:409-424.

Chee MW, Choo WC (2004) Functional imaging of working memory after $24 \mathrm{hr}$ of total sleep deprivation. J Neurosci 24:4560-4567.

Chee MW, Chuah YM (2007) Functional neuroimaging and behavioral correlates of capacity decline in visual short-term memory following sleep deprivation. Proc Natl Acad Sci USA 104:9487-9892.

Chee MW, Soon CS, Lee HL, Pallier C (2004) Left insula activation: a marker for language attainment in bilinguals. Proc Natl Acad Sci USA 101:15265-15270.

Choo WC, Lee WW, Venkatraman V, Sheu FS, Chee MW (2005) Dissociation of cortical regions modulated by both working memory load and sleep deprivation and by sleep deprivation alone. NeuroImage 25:579-587.

Corbetta M, Shulman GL (2002) Control of goal-directed and stimulusdriven attention in the brain. Nat Rev Neurosci 3:201-215.

Corsi-Cabrera M, Arce C, Del Rio-Portilla IY, Perez-Garci E, Guevara MA (1999) Amplitude reduction in visual event-related potentials as a function of sleep deprivation. Sleep 22:181-189.

Datta S, Maclean RR (2007) Neurobiological mechanisms for the regulation of mammalian sleep-wake behavior: reinterpretation of historical evidence and inclusion of contemporary cellular and molecular evidence. Neurosci Biobehav Rev 31:775-824.

Davidson MC, Marrocco RT (2000) Local infusion of scopolamine into intraparietal cortex slows covert orienting in rhesus monkeys. J Neurophysiol 83:1536-1549.

Desimone R, Duncan J (1995) Neural mechanisms of selective visual attention. Annu Rev Neurosci 18:193-222.
Dinges DF (1995) An overview of sleepiness and accidents. J Sleep Res $4: 4-14$.

Dinges DF, Kribbs NB (1991) Performing while sleepy: effects of experimentally-induced sleepiness. In: Sleep, sleepiness and performance (Monk TH, ed), pp 97-128. Chichester, UK: Wiley.

Dinges DF, Pack F, Williams K, Gillen KA, Powell JW, Ott GE, Aptowicz C, Pack AI (1997) Cumulative sleepiness, mood disturbance, and psychomotor vigilance performance decrements during a week of sleep restricted to 4-5 hours per night. Sleep 20:267-277.

Doran SM, Van Dongen HP, Dinges DF (2001) Sustained attention performance during sleep deprivation: evidence of state instability. Arch Ital Biol 139:253-267.

Dorrian J, Rogers NL, Dinges DF (2005) Psychomotor vigilance performance: neurocognitive assay sensitive to sleep loss. In: Sleep deprivation: clinical issues, pharmacology and sleep loss effects (Kushida CA, ed), pp 39-70. New York: Dekker.

Drummond SP, Brown GG, Salamat JS, Gillin JC (2004) Increasing task difficulty facilitates the cerebral compensatory response to total sleep deprivation. Sleep 27:445-451.

Drummond SP, Bischoff-Grethe A, Dinges DF, Ayalon L, Mednick SC, Meloy MJ (2005) The neural basis of the psychomotor vigilance task. Sleep 28:1059-1068

Everitt BJ, Robbins TW (1997) Central cholinergic systems and cognition. Annu Rev Psychol 48:649-684.

Foucher JR, Otzenberger H, Gounot D (2004) Where arousal meets attention: a simultaneous fMRI and EEG recording study. NeuroImage 22:688-697.

Furey ML, Pietrini P, Haxby JV (2000) Cholinergic enhancement and increased selectivity of perceptual processing during working memory. Science 290:2315-2319.

Gray JR, Chabris CF, Braver TS (2003) Neural mechanisms of general fluid intelligence. Nat Neurosci 6:316-322.

Habeck C, Rakitin BC, Moeller J, Scarmeas N, Zarahn E, Brown T, Stern Y (2004) An event-related fMRI study of the neurobehavioral impact of sleep deprivation on performance of a delayed-match-to-sample task. Brain Res Cogn Brain Res 18:306-321.

Hagberg GE, Zito G, Patria F, Sanes JN (2001) Improved detection of eventrelated functional MRI signals using probability functions. NeuroImage 14:1193-1205.

Honey GD, Bullmore ET, Sharma T (2000) Prolonged reaction time to a verbal working memory task predicts increased power of posterior parietal cortical activation. NeuroImage 12:495-503.

Hopfinger JB, Buonocore MH, Mangun GR (2000) The neural mechanisms of top-down attentional control. Nat Neurosci 3:284-291.

Horne JA (1988) Sleep loss and "divergent" thinking ability. Sleep 11:528-536.

Horne JA, Ostberg O (1976) A self-assessment questionnaire to determine morningness-eveningness in human circadian rhythms. Int J Chronobiol 4:97-110.

Horne JA, Reyner LA (1995) Sleep related vehicle accidents. BMJ 310:565-567.

Huang RS, Jung TP, Delorme A, Makeig S (2008) Tonic and phasic electroencephalographic dynamics during continuous compensatory tracking. NeuroImage 39:1896-1909.

Jones BE (2005) Basic mechanisms of sleep-wake states. In: Principles and practice of sleep medicine (Kryger MH, Roth T, Dement WC, eds), pp 136-153. Philadelphia: Elsevier Saunders.

Kastner S, Ungerleider LG (2000) Mechanisms of visual attention in the human cortex. Annu Rev Neurosci 23:315-341.

Kinomura S, Larsson J, Gulyas B, Roland PE (1996) Activation by attention of the human reticular formation and thalamic intralaminar nuclei. Science 271:512-515.

Lim J, Dinges DF (2008) Sleep deprivation and vigilant attention. Ann NY Acad Sci, in press.

Lim J, Choo WC, Chee MW (2007) Reproducibility of changes in behavior and $\mathrm{fMRI}$ activation associated with sleep deprivation in a working memory task. Sleep 30:61-70.

Livingstone M, Hubel D (1981) Effects of sleep and arousal on the processing of visual information in the cat. Nature 291:554-561.

Makeig S, Inlow M (1993) Lapses in alertness: coherence of fluctuations in performance and EEG spectrum. Electroencephalogr Clin Neurophysiol $86: 23-35$. 
Makeig S, Jung TP (1996) Tonic, phasic, and transient EEG correlates of auditory awareness in drowsiness. Brain Res Cogn Brain Res 4:15-25.

Manoach DS, Gollub RL, Benson ES, Searl MM, Goff DC, Halpern E, Saper CB, Rauch SL (2000) Schizophrenic subjects show aberrant fMRI activation of dorsolateral prefrontal cortex and basal ganglia during working memory performance. Biol Psychiatry 48:99-109.

Miezin FM, Maccotta L, Ollinger JM, Petersen SE, Buckner RL (2000) Characterizing the hemodynamic response: effects of presentation rate, sampling procedure, and the possibility of ordering brain activity based on relative timing. NeuroImage 11:735-759.

Mitler MM, Carskadon MA, Czeisler CA, Dement WC, Dinges DF, Graeber RC (1988) Catastrophes, sleep, and public policy: consensus report. Sleep 11:100-109.

Moruzzzi G, Magoun HW (1949) Brain stem reticular formation and activation of the EEG. Electroencephalogr Clin Neurophysiol 1:455-473.

Murphy PC, Sillito AM (1991) Cholinergic enhancement of direction selectivity in the visual cortex of the cat. Neuroscience 40:13-20.

Navon D (1977) Forest before trees: the precedence of global features in visual perception. Cogn Psychol 9:353-383.

O’Connor DH, Fukui MM, Pinsk MA, Kastner S (2002) Attention modulates responses in the human lateral geniculate nucleus. Nat Neurosci 5:1203-1209.

Oken BS, Salinsky MC, Elsas SM (2006) Vigilance, alertness, or sustained attention: physiological basis and measurement. Clin Neurophysiol 117:1885-1901.

Padilla ML, Wood RA, Hale LA, Knight RT (2006) Lapses in a prefrontalextrastriate preparatory attention network predict mistakes. J Cogn Neurosci 18:1477-1487.

Peiris MT, Jones RD, Davidson PR, Carroll GJ, Bones PJ (2006) Frequent lapses of responsiveness during an extended visuomotor tracking task in non-sleep-deprived subjects. J Sleep Res 15:291-300.

Philip P, Akerstedt T (2006) Transport and industrial safety, how are they affected by sleepiness and sleep restriction? Sleep Med Rev 10:347-356.

Phillips JM, McAlonan K, Robb WG, Brown VJ (2000) Cholinergic neurotransmission influences covert orientation of visuospatial attention in the rat. Psychopharmacology (Berl) 150:112-116.

Portas CM, Rees G, Howseman AM, Josephs O, Turner R, Frith CD (1998)
A specific role for the thalamus in mediating the interaction of attention and arousal in humans. J Neurosci 18:8979-8989.

Rypma B, D’Esposito M (1999) The roles of prefrontal brain regions in components of working memory: effects of memory load and interindividual differences. Proc Natl Acad Sci USA 96:6558-6563.

Rypma B, Berger JS, Prabhakaran V, Bly BM, Kimberg DY, Biswal BB, D’Esposito M (2006) Neural correlates of cognitive efficiency. NeuroImage 33:969-979.

Saper CB, Chou TC, Scammell TE (2001) The sleep switch: hypothalamic control of sleep and wakefulness. Trends Neurosci 24:726-731.

Sarter M, Gehring WJ, Kozak R (2006) More attention must be paid: the neurobiology of attentional effort. Brain Res Rev 51:145-160.

Sato H, Hata Y, Masui H, Tsumoto T (1987) A functional role of cholinergic innervation to neurons in the cat visual cortex. J Neurophysiol 58:765-780.

Soon CS, Venkatraman V, Chee MW (2003) Stimulus repetition and hemodynamic response refractoriness in event-related fMRI. Hum Brain Mapp 20:1-12.

Steriade M, McCarley RW (2005) Changing concepts of mechanisms of waking and sleep states. In: Brain control of wakefulness and sleep, pp 1-33. New York: Kluwer Academic.

Szymusiak R, Gvilia I, McGinty D (2007) Hypothalamic control of sleep. Sleep Med 8:291-301.

Uschakov A, Gong H, McGinty D, Szymusiak R (2007) Efferent projections from the median preoptic nucleus to sleep- and arousal-regulatory nuclei in the rat brain. Neuroscience 150:104-120.

Van Dongen HP, Baynard MD, Maislin G, Dinges DF (2004) Systematic interindividual differences in neurobehavioral impairment from sleep loss: evidence of trait-like differential vulnerability. Sleep 27:423-433.

Van Orden KF, Jung TP, Makeig S (2000) Combined eye activity measures accurately estimate changes in sustained visual task performance. Biol Psychol 52:221-240.

Weissman DH, Roberts KC, Visscher KM, Woldorff MG (2006) The neural bases of momentary lapses in attention. Nat Neurosci 9:971-978.

Woldorff MG, Gallen CC, Hampson SA, Hillyard SA, Pantev C, Sobel D, Bloom FE (1993) Modulation of early sensory processing in human auditory cortex during auditory selective attention. Proc Natl Acad Sci USA 90:8722-8726. 